Vol. 76 (2007) [143-154]

\title{
SURJECTIVITY OF LINEAR OPERATORS FROM A BANACH SPACE INTO ITSELF
}

\section{Dimosthenis Drivaliaris and Nikos YanNaKakis}

\begin{abstract}
We show that linear operators from a Banach space into itself which satisfy some relaxed strong accretivity conditions are invertible. Moreover, we characterise a particular class of such operators in the Hilbert space case. By doing so we manage to answer a problem posed by B. Ricceri, concerning a linear second order partial differential operator.
\end{abstract}

\section{INTRODUCTION}

In [5] Saint Raymond proved the following three theorems, which answer a question posed by Ricceri in [3].

TheOREM 1.1. Let $X$ be a real Hilbert space and $A: X \rightarrow X$ be a linear operator. If there exists $c>0$ such that

$$
\langle A x, x\rangle+\|A x\|\|x\| \geqslant c\|x\|^{2}, \text { for all } x \in X,
$$

then $A$ is bounded and invertible.

THEOREM 1.2. Let $X$ be a real Hilbert space and $A: X \rightarrow X$ be a linear operator. If there exist a finite dimensional subspace $L$ of $X$, a bounded projection $P$ from $X$ onto $L, c>0$ and $\gamma>0$ such that,

$$
\langle A x, x\rangle+\|A x\|\|x\|+\gamma\|P A x\|\|x\| \geqslant c\|x\|^{2}, \text { for all } x \in X,
$$

then $A$ is bounded and invertible.

and

THEOREM 1.3. Let $X$ be a real Hilbert space and $A: X \rightarrow X$ be a linear operator. If there exist a compact operator $K: X \rightarrow X$ and $c>0$ such that

$$
\langle A x, x\rangle+\|A x\|\|x\|+\|K A x\|\|x\| \geqslant c\|x\|^{2}, \text { for all } x \in X,
$$

then $A$ is bounded and invertible.

Received 22nd January, 2007

Copyright Clearance Centre, Inc. Serial-fee code: 0004-9727/07 \$A2.00+0.00. 
Conditions (1), (2) and (3) are relaxed coercivity conditions. Therefore Theorems $1.1,1.2$ and 1.3 can be thought of as non-coercive generalisations of the Lax-Milgram Theorem. Note that $(1) \Rightarrow(2) \Rightarrow(3)$.

The class of operators satisfying (1) is strictly larger than that of coercive operators. To see that take any rotation in $\mathbb{R}^{2}$ by an angle $\theta$ larger than $\pi / 2$ and strictly less than $\pi$. Actually all operators $A$ satisfying (1) share a simple geometric property with such rotations: the angle of $x$ and $A x$ is uniformly strictly less than $\pi$ (see [7, Theorem 3.2]).

One can easily see that if an operator $A$ satisfies (1), (2) or (3), then, for some positive constant $\alpha$,

$$
\|A x\| \geqslant \alpha\|x\|, \text { for all } x \in X .
$$

Thus if one additionally assumes that $A$ is positive (or negative like the rotations in $\mathbb{R}^{2}$ of the previous paragraph), then Theorems $1.1,1.2$ and 1.3 become immediate consequences of the well-known fact (see for example [1, Theorem 11.2]) that everywhere defined, positive linear operators which are weakly coercive, that is, which satisfy

$$
\lim _{\|x\| \rightarrow \infty}\|A x\|=\infty
$$

are surjective. Hence the true advantage of Theorems 1.1, 1.2 and 1.3, compared to already existing results, is that $\langle A x, x\rangle$ is allowed to change sign. An example of an operator $A$ satisfying (1) for which $\langle A x, x\rangle$ changes sign can be constructed as follows (see [7]). Let $X=l^{2}(\mathbb{N}),\left\{e_{1}, e_{2}, \ldots\right\}$ be its standard orthonormal basis, $M=\operatorname{span}\left\{e_{1}, e_{2}\right\}$ and $R: M \rightarrow M$ be the rotation by an angle $\pi / 2<\theta<\pi$. Then it is easy to see that the operator $A: X \rightarrow X$ defined by $A\left(x_{1}+x_{2}\right)=R x_{1}+x_{2}$, for all $x_{1} \in M$ and all $x_{2} \in M^{\perp}$, satisfies condition (1) and $\langle A x, x\rangle$ changes sign.

Our main aim in this paper is to study operators from a Banach space into itself which satisfy analogues of conditions (1), (2) and (3).

In the first part of Section 2 we shall show that if a bounded linear operator $A$ satisfies a Banach space version of (1) or (2), then $A$ is invertible. Actually we shall show that this is true even if in (2) we replace the projection $P$ with a finite rank operator $F$. This approach allows us to prove a Banach space analogue of Theorem 1.3 for Banach spaces which have the approximation property. We must note here that since the crucial step in the proof in [5, Theorem 1.2] relies on the Hilbert space structure of $X$ it cannot be generalised to our case (see Remark 2.13). To overcome this difficulty we use an argument based on Banach's Fixed Point Theorem.

In the second part of Section 2 we shall prove that an unbounded linear operator $A$ from a Banach space into itself which satisfies an analogue of condition (1) is invertible if $A+t_{0} I$ is surjective, for some positive $t_{0}$.

Finally, in Section 3 we turn our attention to bounded linear operators on a real Hilbert space which satisfy (1). We show that an operator belongs to this class if and 
only if the intersection of its spectrum with the real line is contained in $[k,+\infty)$, for some $k>0$. That allows us to give an answer to a problem posed by Ricceri in [4, Problem 2].

\section{MAIN RESULTS}

In what follows $X$ is a real Banach space, $\|\cdot\|$ is its norm, $X^{*}$ is its dual space and $\langle\cdot, \cdot\rangle$ is their duality product. By $J: X \rightarrow 2^{X^{*}}$ we shall denote the duality map of $X$ defined by

$$
J(x)=\left\{x^{*} \in X^{*} \mid\left\langle x^{*}, x\right\rangle=\|x\|^{2} \text { and }\left\|x^{*}\right\|=\|x\|\right\} .
$$

Recall that a linear operator $A: D(A) \subseteq X \rightarrow X$ is called accretive if, for each $x \in D(A)$, there exists $x^{*} \in J(x)$ with $\left\langle x^{*}, A x\right\rangle \geqslant 0$. If additionally the operator $A+t_{0} I$ is surjective, for some $t_{0}>0$ (equivalently for all $t_{0}>0$ ), then $A$ is called m-accretive.

As we have already mentioned conditions (1), (2) and (3) are relaxed coercivity conditions. The counterpart of coercivity for maps from a Banach space into itself is strong accretivity:

Definition 2.1: Let $A: D(A) \subseteq X \rightarrow X$ be a linear operator. We say that $A$ is strongly accretive if there exists $c>0$ such that, for each $x \in D(A)$, there exists $x^{*} \in J(x)$ with

$$
\left\langle x^{*}, A x\right\rangle \geqslant c\|x\|^{2} \text {. }
$$

Hence the analogues of conditions (1), (2) and (3) for linear operators from a Banach space into itself can be formulated as follows:

Definition 2.2: Let $A$ be a linear operator from $X$ into itself. We say that $A$ satisfies condition (*) if there exists $c>0$ such that, for each $x \in X$, there exists $x^{*} \in J(x)$ with

$$
\left\langle x^{*}, A x\right\rangle+\|A x\|\|x\| \geqslant c\|x\|^{2} .
$$

Definition 2.3: Let $A$ be a linear operator from $X$ into itself. We say that $A$ satisfies condition (**) if there exist a finite dimensional subspace $L$ of $X$, a bounded projection $P$ from $X$ onto $L, c>0$ and $\gamma>0$ such that, for each $x \in X$, there exists $x^{*} \in J(x)$ with

$$
\left\langle x^{*}, A x\right\rangle+\|A x\|\|x\|+\gamma\|P A x\|\|x\| \geqslant c\|x\|^{2} .
$$

and

Definition 2.4: Let $A$ be a linear operator from $X$ into itself. We say that $A$ satisfies condition $(* * *)$ if there exist a compact operator $K: X \rightarrow X, c>0$ and $\gamma>0$ such that, for each $x \in X$, there exists $x^{*} \in J(x)$ with

$$
\left\langle x^{*}, A x\right\rangle+\|A x\|\|x\|+\gamma\|K A x\|\|x\| \geqslant c\|x\|^{2} .
$$


A class of operators, lying between those satisfying (**) and those satisfying (****) is the following:

Definition 2.5: Let $A$ be a linear operator from $X$ into itself. We say that $A$ satisfies condition $(* * *)$ if there exist a finite rank operator $F: X \rightarrow X, c>0$ and $\gamma>0$ such that, for each $x \in X$, there exists $x^{*} \in J(x)$ with

$$
\left\langle x^{*}, A x\right\rangle+\|A x\|\|x\|+\gamma\|F A x\|\|x\| \geqslant c\|x\|^{2} .
$$

All the conditions defined above are relaxed strong accretivity conditions. It is wellknown that, even nonlinear, continuous strongly accretive operators are surjective (see for example [1, Theorem 13.1]). We shall prove that bounded linear operators satisfying one of $(*),(* *),(* * *)$ and $(* * * *)$ are also surjective (to be precise, for the last case we shall additionally assume that $X$ has the approximation property).

We start with operators satisfying $(*),(* *)$ and $(* * *)$. Since $(*)$ and $(* *)$ are special cases of $(* * *)$ we shall only deal with the latter.

LEMMA 2.6. Let $A$ be a linear operator satisfying condition (***). Then, for all $x \in X$,

$$
\|A x\| \geqslant \frac{c}{2+\gamma\|F\|}\|x\| .
$$

Proof: If we take $x \in X$, then, for the $x^{*} \in J(x)$ corresponding to $x$ by $(* * *)$, we have that

$$
c\|x\|^{2} \leqslant\left\langle x^{*}, A x\right\rangle+\|A x\|\|x\|+\gamma\|F A x\|\|x\| \leqslant(2+\gamma\|F\|)\|A x\|\|x\|
$$

and the result follows immediately.

For the rest of the section we shall denote $\operatorname{Ker}(F)$ by $M$. Since $F$ is a finite rank operator, $M$ has finite codimension. Thus there exists a finite dimensional subspace $N$ of $X$ such that $X=M \oplus N$.

We continue by showing that if a linear operator $A$ satisfies condition (***), then, for all $t>0, A+t I$ satisfies condition (***) on $M$.

LemMA 2.7. Let $A$ be a linear operator satisfying condition (***). Then, for each $x \in M$, there exists $x^{*} \in J(x)$ such that, for all $t>0$,

$$
\left\langle x^{*},(A+t I) x\right\rangle+\|(A+t I) x\|\|x\|+\delta\|F(A+t I) x\|\|x\| \geqslant c\|x\|^{2} .
$$

Proof: Let $t>0$. If $x \in M$, then $F x=0$. Thus if $x^{*} \in J(x)$ is the one corresponding by $(* * *)$ to $x$, we have that

$$
\begin{aligned}
\left\langle x^{*}, A x+t x\right\rangle+\| & (A+t I) x\|\| x\|+\gamma\| F(A+t I) x\|\| x \| \\
& \geqslant\left\langle x^{*}, A x\right\rangle+t\|x\|^{2}+(\|A x\|-t\|x\|)\|x\|+\gamma\|F A x\|\|x\| \\
& =\left\langle x^{*}, A x\right\rangle+\|A x\|\|x\|+\gamma\|F A x\|\|x\| \\
& \geqslant c\|x\|^{2}
\end{aligned}
$$


where the last inequality follows from (***).

Combining Lemma 2.6 and Lemma 2.7 we get:

LEMmA 2.8. Let $A$ be a linear operator satisfying condition (***). Then, for all $x \in M$ and all $t>0$,

$$
\|(A+t I) x\| \geqslant \frac{c}{2+\gamma\|F\|}\|x\| .
$$

Now assume that $A$ is bounded. It is immediate from Lemma 2.6 that $A$ has closed range. Moreover from Lemma 2.8 we have that, for all $t>0,(A+t I)(M)$ is closed. Since $N$ is finite dimensional, $(A+t I)(N)$ is finite dimensional and hence

$$
(A+t I)(X)=(A+t I)(M)+(A+t I)(N)
$$

is closed. So we have:

Lemma 2.9. Let $A$ be a bounded linear operator satisfying condition (***). Then $A+t I$ has closed range, for all $t \geqslant 0$.

For the proof of the next proposition, which is the crucial step towards the proof of the surjectivity of bounded linear operators satisfying $(* * *)$, we shall need the following lemma about the norm of the projections onto a finite codimensional subspace of a Banach space.

Lemma 2.10. Let $X$ be a Banach space. If $M$ is a closed subspace of $X$ with codimension $n$, then there exists a projection $Q$ such that $\operatorname{Ker}(Q)=M$ and $\|Q\| \leqslant 2 n$.

Proof: We are going to prove the result by induction. For $\operatorname{codim}(M)=1$ it is easy to construct a projection $Q$ such that $\operatorname{Ker}(Q)=M$ and $\|Q\| \leqslant 2$.

Now assume that for any Banach space $X$ and any subspace $M$ of $X$ with $\operatorname{codim}(M)=n-1$ there exists a bounded projection $Q: X \rightarrow X$ with $\operatorname{Ker}(Q)=M$ and $\|Q\| \leqslant 2(n-1)$.

Let $X$ be a Banach space and $M$ be a subspace of $X$ with $\operatorname{codim}(M)=n$. Then there exist $x_{1}, x_{2}, \ldots, x_{n} \in X$ linearly independent with

$$
X=M \oplus \operatorname{span}\left\{x_{1}, x_{2}, \ldots, x_{n}\right\} .
$$

Let $M_{1}=M \oplus \operatorname{span}\left\{x_{1}\right\}$. Then obviously $\operatorname{codim}\left(M_{1}\right)=n-1$. Therefore, by the inductive hypothesis, there exists a bounded projection $Q_{1}$ with $\operatorname{Ker}\left(Q_{1}\right)=M_{1}$ and $\left\|Q_{1}\right\| \leqslant 2(n-1)$. Now let $M_{2}=M \oplus Q_{1}(X)$. It is obvious that $\operatorname{codim}\left(M_{2}\right)=1$. Therefore, by the base step of the induction, there exists a bounded projection $Q_{2}$ with $\operatorname{Ker}\left(Q_{2}\right)=M_{2}$ and $\left\|Q_{2}\right\| \leqslant 2$. If $Q=Q_{1}+Q_{2}$, then it is easy to see that $Q$ is a projection with $\operatorname{Ker}(Q)=M$ and $\|Q\| \leqslant 2 n$.

REMARK 2.11. Note that the previous lemma is an immediate consequence of [6, Corollary 11, p. 117]. The reason we give the above proof is that, since our main interest is the existence of such a projection and not a sharp estimate of its norm, it is much simpler. 
Proposition 2.12. Let $A$ be a bounded linear operator satisfying (***). If, for some $t_{0} \geqslant 0$, the subspace $\left(A+t_{0} I\right)(M)$ has codimension $n$, then so does the subspace $(A+t I)(M)$, for all $t$ with $\left|t-t_{0}\right|<\delta$, where $\delta=c /((2+\gamma\|F\|)(1+2 n))$.

Proof: Since

$$
\operatorname{codim}\left(\left(A+t_{0} I\right)(M)\right)=n
$$

by Lemma 2.10, we can find a projection $Q$ such that $\operatorname{Ker}(Q)=\left(A+t_{0} I\right)(M)$ and $\|Q\| \leqslant 2 n$.

Let $y \in X$. Then, for any $z \in X$, there exists a unique $x \in M$ such that

$$
y+\left(t_{0}-t\right) z=\left(A+t_{0} I\right) x+Q\left(y+\left(t_{0}-t\right) z\right) .
$$

Hence we can define a map $T: X \rightarrow X$ with $T z=x$. We shall show that $T$ is a contraction. To this end let $z_{1}, z_{2} \in X$. From the above, Lemma 2.8 and the hypothesis about $t$ we get

$$
\begin{aligned}
\left\|T z_{1}-T z_{2}\right\|=\left\|x_{1}-x_{2}\right\| & \leqslant \frac{2+\gamma\|F\|}{c}\left\|\left(A+t_{0} I\right)\left(x_{1}-x_{2}\right)\right\| \\
& \leqslant \frac{2+\gamma\|F\|}{c}\left|t-t_{0}\right|\|I-Q\|\left\|z_{1}-z_{2}\right\| \\
& \leqslant \frac{2+\gamma\|F\|}{c}\left|t-t_{0}\right|(1+\|Q\|)\left\|z_{1}-z_{2}\right\| \\
& \leqslant \frac{(2+\gamma\|F\|)(1+2 n)}{c}\left|t-t_{0}\right|\left\|z_{1}-z_{2}\right\| \\
& <\left\|z_{1}-z_{2}\right\| .
\end{aligned}
$$

By Banach's Fixed Point Theorem, $T$ has a unique fixed point, that is, there exists a unique $x \in X$ such that

$$
T x=x .
$$

Since $T$ takes its values in $M$, we get that there exists a unique $x \in M$ such that

$$
y=(A+t I) x+Q\left(y+\left(t_{0}-t\right) x\right) .
$$

Since $y$ was arbitrary and the representation is unique we get that

$$
X=(A+t I)(M) \oplus Q(X)
$$

and hence

$$
\operatorname{codim}((A+t I)(M))=n .
$$

REMARK 2.13. We should note here that the above proof is significantly different from the corresponding one in Saint Raymond's paper [5, Lemma 7]. The reason is that a basic element in his proof is the existence of the orthogonal complement of $(A+t I)(M)$. In the Banach space setting one cannot replace this orthogonal complement with an arbitrary direct summand unless $(A+t I)(M)$ is a complemented subspace, which is not a priori known. 
We are now ready for our main result in the bounded case. Without loss of generality we may assume that $\operatorname{codim}(M)=\operatorname{dim}(N)=n$.

THEOREM 2.14. Let $A$ be a bounded linear operator satisfying (***). Then $A$ is invertible.

Proof: The injectivity of $A$ follows from Lemma 2.6. We shall show that $A$ is onto. For $t_{0}>\|A\|, A+t_{0} I$ is invertible and thus

$$
\operatorname{codim}\left(\left(A+t_{0} I\right)(M)\right)=n .
$$

Now we can use Proposition 2.12 and get that $\operatorname{codim}((A+t I)(M))=n$, for any $t$ with $\left|t-t_{0}\right|<\delta$. By repeating this procedure ( $\delta$ is independent of $t$ ) we get that

$$
\operatorname{codim}(A(M))=n .
$$

By the injectivity of $A$ we get that $A(M) \cap A(N)=\{0\}$ and $\operatorname{dim}(A(N))=n$. Since $A(X)=A(M)+A(N)$ and, by Lemma 2.9, the range of $A$ is closed we get that $A(X)=X$ and so $A$ is onto.

As we have already mentioned if a linear operator satisfies condition $(*)$ or condition $(* *)$, then it obviously satisfies (***) and so we have:

COROLlary 2.15. Let $A$ be a bounded linear operator satisfying (*) or (**). Then $A$ is invertible.

We continue by proving that bounded linear operators satisfying condition (****) are also invertible if $X$ has the approximation property. Recall that a Banach space $X$ has the approximation property if every compact operator on $X$ is the limit of a sequence of finite rank operators.

THEOREM 2.16. Let $X$ be a Banach space with the approximation property and $A$ be a bounded linear operator satisfying (****). Then $A$ is invertible.

Proof: Since $X$ has the approximation property and $K$ is compact, for any $\varepsilon>0$, there exists a finite rank operator $F$ such that $\|K-F\|<\varepsilon$. Hence

$$
\|K x\|-\varepsilon\|x\|<\|F x\| \text {, for all } x \in X .
$$

The above inequality and (****) imply that, for all $x \in X$,

$$
\begin{aligned}
\left\langle x^{*}, A x\right\rangle+\|A x\|\|x\|+\|F A x\|\|x\| & >\left\langle x^{*}, A x\right\rangle+\|A x\|\|x\|+\|K A x\|\|x\|-\varepsilon\|A x\|\|x\| \\
& >\left\langle x^{*}, A x\right\rangle+\|A x\|\|x\|+\|K A x\|\|x\|-\varepsilon\|A\|\|x\|^{2} \\
& \geqslant(c-\varepsilon\|A\|)\|x\|^{2} .
\end{aligned}
$$

For $\varepsilon<c /\|A\|$ that immediately implies that $A$ satisfies (***). So, by Theorem 2.14, $A$ is invertible. 
It is straightforward to see that the above results also hold for complex Banach spaces provided that in the conditions $(*),(* *),(* * *)$ and $(* * * *)$ instead of $\left\langle x^{*}, A x\right\rangle$ we use $\operatorname{Re}\left\langle x^{*}, A x\right\rangle$.

A natural question that arises is whether conditions $(*),(* *),(* * *)$ and $(* * * *)$ can imply, as in Saint Raymond's results, that $A$ is automatically bounded. In general the answer will be no, since not even strongly accretive operators are necessarily bounded. On the other hand under certain assumptions on $X$ (for example, if $X$ is uniformly convex) linear accretive operators are bounded (see [2, Theorem 7.50, p. 397]). So we can reformulate the question as follows: Under which assumptions on $X$ is a linear operator satisfying one of conditions $(*),(* *),(* * *)$ and $(* * * *)$ automatically bounded?

For the rest of this section we shall deal with unbounded operators

$$
A: D(A) \subseteq X \rightarrow X
$$

We shall show that if such an operator $A$ satisfies condition (*) (the only thing that we need to change in the definition is that the condition must hold for all $x \in D(A)$ ) and, for some $t_{0}>0$, the operator $A+t_{0} I$ is surjective, then $A$ is invertible. Note that this additional hypothesis is exactly the same with the one of $m$-accretivity.

We start with a lemma analogous to Lemmas 2.6 and 2.8 .

LEMMA 2.17. Let $A: D(A) \subseteq X \rightarrow X$ be a linear operator satisfying condition (*). Then, for all $x \in D(A)$ and all $t \geqslant 0$,

$$
\|(A+t I) x\| \geqslant \frac{c}{2}\|x\| .
$$

We can now state and prove the main result for the unbounded case.

THEOREM 2.18. Let $A: D(A) \subseteq X \rightarrow X$ be a linear operator satisfying (*). If $A+t_{0} I$ is surjective, for some $t_{0} \geqslant 0$, then $A$ is invertible.

Proof: By the previous lemma we get that $A$ is injective. Let $y \in X$. Then, for any $z \in X$, there exists a unique $x \in D(A)$ such that

$$
y+\left(t_{0}-t\right) z=\left(A+t_{0} I\right) x
$$

Hence as in the proof of Theorem 2.14 we can define $T: X \rightarrow X$ by $T z=x$ and use Lemma 2.17 to show that $T$ is a contraction. So, by Banach's Fixed Point Theorem, there exists a unique $x \in D(A)$ such that $T x=x$. Thus the operator $A+t I$ is also surjective, for all $t$ with $\left|t-t_{0}\right|<\delta=c / 2$. Continuing this procedure (again $\delta$ is independent of $t$ ) we get that $A$ is surjective. Using again the previous lemma we also get that the inverse of $A$ is bounded.

If both $X$ and $X^{*}$ are uniformly convex then, not necessarily linear, weakly coercive and $m$-accretive operators are surjective (see [1, Theorem 13.4]). Theorem 2.18 allows us to get an easy proof of that result (without any restrictions on $X$ ) for linear operators. 
Corollary 2.19. Let $A: D(A) \subseteq X \rightarrow X$ be a linear, weakly coercive, $m$ accretive operator. Then $A$ is invertible.

Proof: Obviously, since $A$ is linear, its weak coercivity implies that there exists $c>0$ such that $\|A x\| \geqslant c\|x\|$, for all $x \in X$. Combining that with the accretivity of $A$ we immediately get that $A$ satisfies (*). Since the m-accretivity of $A$ implies that $A+t_{0} I$ is surjective, for some $t_{0} \geqslant 0$, the result follows from Theorem 2.18.

Note that again the results also hold for complex Banach spaces if we replace $\left\langle x^{*}, A x\right\rangle$ with $\operatorname{Re}\left\langle x^{*}, A x\right\rangle$.

Recall that, as we have already mentioned in the Introduction, the main advantage of Theorems 1.1, 1.2 and 1.3, compared to other existing surjectivity results, is that $\langle A x, x\rangle$ is allowed to change sign. This is the case in the next simple example of a differential operator $A$ which satisfies the hypotheses of Theorem 2.18 .

EXAMPLE 2.20. Let $1<p<\infty$ and $\lambda \in \mathbb{R}$ with $\lambda^{2}<1 / 2$ and let

$$
A: D(A) \subseteq L^{p}(0,1) \rightarrow L^{p}(0,1)
$$

with

$$
D(A)=\left\{x \in W^{1, p}(0,1) \mid x(0)=0\right\}
$$

defined by

$$
A x=x^{\prime}-\lambda^{2} x, \text { for all } x \in D(A) .
$$

Denote the usual norm of $L^{p}(0,1)$ by $\|\cdot\|$. Note that $\left\|x^{\prime}\right\| \geqslant\|x\|$, for any $x \in D(A)$. With this norm the duality map of $L^{p}(0,1)$ is single valued. In particular, for all $x \in$ $L^{p}(0,1)$,

$$
J(x)=\frac{x|x|^{p-2}}{\|x\|^{p-2}}
$$

Hence we have that

$$
\langle J(x), A x\rangle=\frac{1}{p\|x\|^{p-2}}|x(1)|^{p}-\lambda^{2}\|x\|^{2}
$$

and obviously $\langle J(x), A x\rangle$ may change sign.

On the other hand

$$
\|A x\| \geqslant\left(1-\lambda^{2}\right)\|x\|
$$

Thus

$$
\begin{aligned}
\langle J(x), A x\rangle+\|A x\|\|x\| & \geqslant \frac{1}{p\|x\|^{p-2}}|x(1)|^{p}+\left(1-2 \lambda^{2}\right)\|x\|^{2} \\
& \geqslant c\|x\|^{2},
\end{aligned}
$$

with $c=1-2 \lambda^{2}>0$.

Finally, it is known that the Cauchy problem

$$
x^{\prime}=f, x(0)=0
$$


has a unique solution $x \in D(A)$, for any $f \in L^{p}(0,1)$. Hence for $t_{0}=\lambda^{2}$ the operator $A+t_{0} I$ is surjective. Thus $A$ satisfies the hypotheses of Theorem 2.18 .

\section{Characterisations of operators satisfying CONDition (1).}

In this section we shall discuss bounded linear operators on a real Hilbert space which satisfy condition (1). By $\sigma(A)$ we denote the spectrum of an operator $A$.

Proposition 3.1. Let $X$ be a real Hilbert space and $A: X \rightarrow X$ be a bounded linear operator. The following are equivalent:

(i) A satisfies condition (1).

(ii) (a) There exists $\alpha>0$ such that $\|A x\| \geqslant \alpha\|x\|$, for all $x \in X$.

(b) There exists $\beta>-1$ such that

$$
\frac{\langle A x, x\rangle}{\|A x\|\|x\|} \geqslant \beta, \text { for all } x \neq 0 \text {. }
$$

(iii) There exists $L>0$ such that $\|A x+t x\| \geqslant L\|x\|$, for all $x \in X$ and all $t \geqslant 0$.

(iv) $A+t I$ is invertible, for all $t \geqslant 0$.

(v) There exists $k>0$ such that $\sigma(A) \cap \mathbb{R} \subseteq[k,+\infty)$.

Proof: (i) $\Leftrightarrow$ (ii): See [7, Theorem 3.2].

(i) $\Rightarrow$ (iii): This is [5, Lemma 5] (with $V=\{0\}$ ).

(iii) $\Rightarrow$ (iv): This is [5, Lemma 7] (again with $V=\{0\}$ ).

(iv) $\Rightarrow$ (iii): Assume that (iii) does not hold. Then there exist a sequence of positive real numbers $\left\{t_{n}\right\}_{n \in \mathbb{N}}$ and a sequence $\left\{x_{n}\right\}_{n \in \mathbb{N}}$ of elements of $X$ with $\left\|x_{n}\right\|=1$ such that

$$
\left\|\left(A+t_{n} I\right) x_{n}\right\| \leqslant \frac{1}{n}
$$

for all $n \in \mathbb{N}$. It is obvious that $\left\{t_{n}\right\}_{n \in \mathbb{N}}$ is bounded by $\|A\|+1$ and hence, without loss of generality, we may assume that there exists $t_{0} \geqslant 0$ such that $\lim t_{n}=t_{0}$ as $n \rightarrow \infty$. But then, since

$$
\left\|\left(A+t_{0} I\right) x_{n}\right\| \leqslant\left\|\left(A+t_{n} I\right) x_{n}\right\|+\left|t_{0}-t_{n}\right|
$$

we have

$$
\lim _{n \rightarrow \infty}\left\|\left(A+t_{0} I\right) x_{n}\right\|=0
$$

which is a contradiction since $A+t_{0} I$ is invertible.

(iv) $\Leftrightarrow(\mathrm{v})$ : Obvious.

(iii) $\Rightarrow$ (i): Assume that (i) does not hold. Then there exists a sequence $\left\{x_{n}\right\}_{n \in N}$ of elements of $X$ with $\left\|x_{n}\right\|=1$ and

$$
0 \leqslant\left\langle A x_{n}, x_{n}\right\rangle+\left\|A x_{n}\right\| \leqslant \frac{1}{n}
$$


for all $n \in \mathbb{N}$. By (iii) and since $A$ is bounded we have that

$$
-\|A\| \leqslant\left\langle A x_{n}, x_{n}\right\rangle \leqslant \frac{1}{n}-L,
$$

for all $n \in \mathbb{N}$. Hence by passing to a subsequence, which for simplicity we denote again by $\left\{x_{n}\right\}_{n \in \mathbb{N}}$, we get that there exists $t_{0}>0$ such that

$$
\lim _{n \rightarrow \infty}\left\langle A x_{n}, x_{n}\right\rangle=-t_{0}<0
$$

Using again (4) we also get that $\lim \left\|A x_{n}\right\|=t_{0}$ as $n \rightarrow \infty$. Therefore we have that

$$
\left\|\left(A+t_{0} I\right) x_{n}\right\|^{2}=\left\|A x_{n}\right\|^{2}+2 t_{0}\left\langle A x_{n}, x_{n}\right\rangle+t_{0}^{2} .
$$

By letting $n \rightarrow \infty$ we get that $\lim \left\|\left(A+t_{0} I\right) x_{n}\right\|=0$ which is obviously a contradiction.

REMARK 3.2. For finite dimensional $X$ the equivalence $(i) \Leftrightarrow(v)$ was proved in $[7$, Theorem 3.1].

The next proposition shows that if we additionally assume that $A$ is symmetric, then condition (1) is equivalent to $A$ being coercive.

Proposition 3.3. Let $X$ be a real Hilbert space and $A: X \rightarrow X$ be a symmetric operator. Then $A$ satisfies (1) if and only if $A$ is coercive.

Proof: It is obvious that any coercive operator satisfies (1). Conversely assume that $A$ satisfies (1). By Proposition 3.1 we get that

$$
\sigma(A) \cap \mathbb{R} \subseteq[k,+\infty),
$$

for some $k>0$. Thus, since $A$ is symmetric,

$$
k \leqslant \inf _{\|x\|=1}\langle A x, x\rangle
$$

and hence $A$ is coercive.

Using the above proposition we can give an answer to a problem posed by Ricceri in [4, Problem 2]:

Problem 3.4. Let $\Omega$ be a bounded open set in $\mathbb{R}^{n}$ and let $a \in L^{\infty}(\Omega)$. Consider $H_{0}^{1}(\Omega)$ with its usual norm. Find necessary and sufficient conditions in order that the quantity

$$
\inf _{\|u\|=1}\left\{\int_{\Omega}\left(|\nabla u|^{2}+a(x)|u|^{2}\right) d x+\sup _{\|v\|=1}\left(\int_{\Omega}(\nabla u \nabla v+a(x) u v) d x\right)\right\}
$$

is strictly positive.

We have the following: 
Proposition 3.5. If $\Omega$ and $a$ are as above then (5) is strictly positive if and only if the quantity

$$
\inf _{\|u\|=1}\left\{\int_{\Omega}\left(|\nabla u|^{2}+a(x)|u|^{2}\right) d x\right\}
$$

is strictly positive.

PRoOF: Let $A: H_{0}^{1}(\Omega) \rightarrow H_{0}^{1}(\Omega)$ be the operator defined by

$$
\langle A u, v\rangle=\int_{\Omega}(\nabla u \nabla v+a(x) u v) d x, \text { for all } u, v \in H_{0}^{1}(\Omega) .
$$

Obviously $A$ is symmetric. It is immediate that the quantity (5) is strictly positive if and only if $A$ satisfies (1). But since $A$ is symmetric, by Proposition 3.3, it satisfies (1) if and only if it is coercive. Hence (5) is strictly positive if and only if the quantity

$$
\inf _{\|u\|=1}\left\{\int_{\Omega}\left(|\nabla u|^{2}+a(x)|u|^{2}\right) d x\right\}
$$

is strictly positive.

\section{REFERENCES}

[1] K. Deimling, Nonlinear functional analysis (Springer-Verlag, Berlin, 1985).

[2] S. Hu and N. S. Papageorgiou, Handbook of multivalued analysis, Vol. I (Kluwer Academic Publishers, Dordrecht, 1997).

[3] B. Ricceri, 'Existence theorems for nonlinear problems', Rend. Accad. Naz. Sci. XL Mem. Mat. (5) 11 (1987), 77-99.

[4] B. Ricceri, 'On some motivated conjectures and problems', Mathematiche (Catania) 51 (1996), 369-373.

[5] J. Saint Raymond, 'A generalization of Lax-Milgram's theorem', Matematiche (Catania) 52 (1997), 149-157.

[6] P. Wojtaszczyk, Banach spaces for analysts (Cambridge University Press, Cambridge, 1991).

[7] N.D. Yen and B.T. Kim, 'Linear operators satisfying the assumptions of some generalized Lax-Milgram theorems', Acta Math. Vietnam. 26 (2001), 407-417.

Department of Financial and Management Engineering

University of the Aegean

31, Fostini Str.

82100 Chios

Greece

e-mail: d.drivaliaris@fme.aegean.gr
Department of Mathematics

School of Applied Mathematics and Natural Sciences

National Technical University of Athens Iroon Polytexneiou 9

15780 Zografou

Greece

e-mail: nyian@math.ntua.gr 